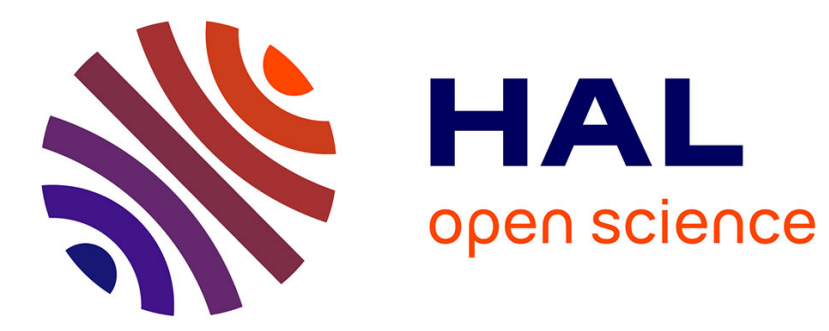

\title{
Measuring routing tables in the internet
}

Elie Rotenberg, Christophe Crespelle, Matthieu Latapy

\section{To cite this version:}

Elie Rotenberg, Christophe Crespelle, Matthieu Latapy. Measuring routing tables in the internet. The Sixth IEEE International Workshop on Network Science for Communication Networks, Apr 2014, Toronto, Canada. pp.795-800, 10.1109/INFCOMW.2014.6849332 . hal-01211167

\section{HAL Id: hal-01211167 https://hal.science/hal-01211167}

Submitted on 3 Oct 2015

HAL is a multi-disciplinary open access archive for the deposit and dissemination of scientific research documents, whether they are published or not. The documents may come from teaching and research institutions in France or abroad, or from public or private research centers.
L'archive ouverte pluridisciplinaire HAL, est destinée au dépôt et à la diffusion de documents scientifiques de niveau recherche, publiés ou non, émanant des établissements d'enseignement et de recherche français ou étrangers, des laboratoires publics ou privés. 


\section{Measuring Routing Tables in the Internet}

\author{
Élie Rotenberg \\ UPMC Univ Paris 6, LIP6 \\ CNRS, ENS de Lyon \\ Elie.Rotenberg@lip6.fr
}

\author{
Christophe Crespelle \\ Université Claude Bernard Lyon 1, DANTE/INRIA \\ LIP, CNRS, ENS de Lyon, Université de Lyon \\ Christophe.Crespelle@inria.fr
}

\author{
Matthieu Latapy \\ UPMC Univ Paris 6, LIP6 \\ CNRS \\ Matthieu.Latapy@lip6.fr
}

\begin{abstract}
The most basic function of an Internet router is to decide, for a given packet, which of its interfaces it will use to forward it to its next hop. To do so, routers maintain a routing table, in which they look up for a prefix of the destination address. The routing table associates an interface of the router to this prefix, and this interface is used to forward the packet. We explore here a new measurement method based upon distributed UDP probing to estimate this routing table for Internet routers.
\end{abstract}

\section{INTRODUCTION}

The role of Internet routers is to forward packets locally to ensure that at the global scope, the packets traveling through the network will reach their destinations. The routing heuristics are diverse, but the result of routing itself can always be seen as a collection of pairs of a packet, and an interface of the router, which it uses to pass the packet to its gateway for its next hop.

However, the details of how this interface is chosen are diverse, and generally not publicly disclosed. The exact nature of the decision leading to the choice of a particular interface for a given packet can depend on multiple factors, including the destination address prefix, the AS of the destination, the packet IP identifier, static configuration, random or pseudorandom load-balancing factors, and more, implementing the routing policy of the router. In its most general definition, a routing table of a router $r$ is a set of rules that design which interfaces of $r$ should be used to send or forward a message towards a given destination. It is a set of rules where each rule $D \rightarrow I$ indicates that for any given destination $d \in D$, an interface $i \in I$ should be used by $r$ to send a message towards $d$. The sets $D$ of destination are either included one in another or disjoint for consistency. (In practice, each $D$ is often a set of destination addresses matching a certain binary prefix)

The knowledge of the actual routing tables, resulting from both static and dynamic configuration, is critical for understanding and modelling routing in the Internet topologies. They define the local behavior of the routers from which the global behavior of the network emerges.

We present here a measurement method that allows to estimate partially or totally such "routing tables". We use a measurement primitive, UDP Ping, to measure the interface used by a target router to route traffic back towards a given monitor source (Section II-A). This primitive is used repeatedly from a large amount of distribution monitors to gather information (Section II-B). This information is then processed into constraints on the rules of the routing table
(Section III). Several assumptions may then be used to further infer these rules, estimating more practically the possible routing table of the target routers (Section IV). We finally assess the principle of this method by conducting a series of practical measurements (Section V).

\section{Measurement Method}

\section{A. UDP Ping}

UDP Ping is a measurement primitive inspired by IP aliasing techniques that we have developped in the context of router degree measurement [1], which allows to discover the interfaces used by a target router to send messages towards monitors that we control.

Let $t$ be an IP adress which we call the target, and $r(t)$ the node (router or end-host) to which $t$ belongs. RFCs [2] and [3] state that when a monitor $m$ sends an UDP packet with destination $t$ on an unallocated port, then $r(t)$ should answer with and ICMP Destination Unreachable packet to $m$. An important detail is that the source of this ICMP packet is in principle the IP address of the interface $i$ used by $r(t)$ to send packets towards $m$. (See Fig. 1)

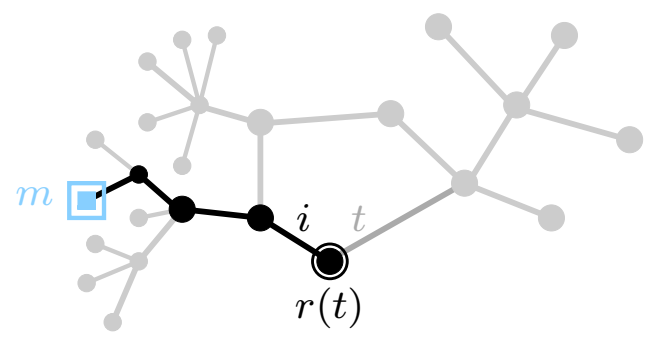

Fig. 1. Monitor $m$ sends a UDP packet with destination address $t$ on an unallocated port; the node $r(t)$ answers with an ICMP packet with source address $i$, and thus $m$ discovers interface $i$ of $r(t)$.

We have studied extensively UDP Ping in a previous work [1]. We concluded that when $r(t)$ properly implements the RFCs (which we can detect for a given router), then it allows to reliably discover its interfaces. A single run of UDP Ping from a monitor $m$ leads to the observation of an interface $i$ used by $r(t)$ to route towards $m$. However, $r(t)$ may not always use $i$ to route towards $m$. To capture all such interfaces, we use UDP Ping repeatedly to observe all of them. The set $m(t)=\left\{i_{1}, i_{2}, \ldots\right\}$ constructed by repeatedly probing $r(t)$ from $m$ is the set of all the interfaces that $r(t)$ uses to route towards $m$. 


\section{B. Distributed UDP Ping}

While UDP Ping itself only provides information on the interfaces used by a target to route towards a given monitor, it can be used distributedly to gather complete information depending on the quality of the monitor set. As explained in [4], the distributed usage of UDP Ping from a monitor set that is large enough and well distributed in the Internet allows to discover all the network interfaces of Internet core routers. Instead, border interfaces would be very hard to observe.

Depending on the configuration of the target, the topological meaning of "well distributed", i.e. "leading to the inference of many rules", could be well distributed in the IPv4 adressing space, or in ASes.

Given a monitor set $M=\left\{m_{1}, m_{2}, \ldots\right\}$, using UDP Ping from each monitor towards a target $t$ leads to the observation of a set $M(t)=\{(m, m(t))\}$, where $m$ is an IP address and $m(t)$ is the set of interfaces of $r(t)$ used to forwards packets towards $m$.

\section{CONSTRAINTS OBTAINED FROM MEASUREMENT}

The routing tables of routers have structural specificites (Section III-A) that allows us to use the results of the measurement method described in Section II-A to deduct constraints on the routing table of a given target router (Section III-B).

\section{A. Structure of the rules}

As presented in Section I, the routing table of a router $r$ is composed of a list of rules $\left\{D_{k} \rightarrow I_{k}\right\}$, where $D_{k}$ is a set of destination addresses, and $I_{k}$ is the set of interfaces used by $r$ to route towards the destinations in $D_{k}$.

By design, routing tables share a number of structural properties resulting from basic optimization concerns. (1) the interface sets are minimal: each interface in a given $I_{k}$ is actually used to route towards each destination in $D_{k}$ (no "unused" interface in $D_{k}$ ). (2) two destination sets $D_{k}$ and $D_{k^{\prime}}$ are either included one in another, or disjoint, so that the mostspecific destination set lookup for a given destination is fast. (3) thanks to the very high practictal efficiency of dedicated hardware, each $D_{k}$ is usually a prefix class: there exist a binary prefix $p_{k}$ of length $n_{k}$ such that $D_{k}$ is exactly the set of IP addresses that match $p_{k}$. We then denote $D_{k}=p_{k} / n_{k}$. In this form, the rules can be conveniently represented in an actual table (See Fig. 2), hence the name "routing table". (4) as a consequence of (2) and (3), there can not be two rules $p .0 \rightarrow I$ and $p .1 \rightarrow I$ : they would be replaced by a single, equivalent rule $p \rightarrow I$.

\section{B. Constraints from Distributed UDP Ping}

The results from Distributed UDP Ping from a monitor set $M$ towards a router $r(t)$ (Section II) can be interpreted in terms of rules of the routing table of $r$.

Distributed UDP Ping outputs a list $M(t)=\{(m, m(t)\}$ where each $m$ is an IP address and $m(t)$ is the set of all the interfaces of $r(t)$ uses to route towards $m$. This means that for any rule $D_{k} \rightarrow I_{k}$ in the routing table of $r(t)$ such that $m \in D_{k}$, then each interface in $m(t)$ is also in $I_{k}$, i.e.

\begin{tabular}{|l|l|l|}
\hline Rule $k$ & Destination prefix $p / n$ & Exit interface(s) $I$ \\
\hline 1 & $128.32 .0 .0 / 13$ & 83.238 .96 .26 \\
\hline 2 & $128.40 .0 .0 / 13$ & 195.114 .175 .54 \\
\hline 3 & $128.112 .139 .64 / 26$ & 83.238 .96 .26 \\
\hline 4 & $128.112 .139 .0 / 26$ & 83.238 .96 .26$, \\
& & 195.114 .175 .54 \\
\hline 5 & $128.114 .63 .0 / 26$ & 83.238 .96 .26$, \\
& & 195.114 .175 .54 \\
\hline$\ldots$ & $\ldots$ & $\ldots$ \\
\hline
\end{tabular}

Fig. 2. Example of a routing table. The router has two interfaces, 83.238.96.26 and 195.114.175.54. If the router needs to route a packet, it choses the longest matching prefix from its table and forwards it to the next gateway through one of the exit interfaces. Rule 1 matches a prefix of length 13. Rules 4 and 5 show examples of multiple exit interfaces configurations, probably implementing a form of load-balanding.

$m(t) \subseteq I_{k}$. Conversely, since all the interfaces used by $r(t)$ to route towards $m$ are in $m(t)$, then $I_{k} \subseteq m(t)$. In terms of prefixes, this means that there must exist a prefix $p_{m} / n_{m}$ such that $m$ matches $p_{m}, p_{m} / n_{m} \rightarrow m(t)$, but also that for each $m^{\prime}$ also matching $p_{m}$, then $m(t)=m^{\prime}(t)$.

Therefore, the constraints deducted from the observation from each monitor $m$ are:

- There must exist a rule $p / n \rightarrow m(t)$ such that $m$ matches p. (Existence constraint)

- For each rule $p^{\prime} / n^{\prime} \rightarrow I$ such that $m$ matches $p^{\prime}$, then $I=m(t)$. (Consistence constraint)

Note that the constraints deducted from the measurement largely depend on the nature of the monitor set $M$. For exemple, let us assume that two monitors $m_{0}$ and $m_{1}$ are such that $m_{1}(t) \neq m_{2}(t)$, and their longest common prefix is $p$, such that $m_{0}$ matches $p .0$ and $m_{1}$ matches $p .1$. Then their can be no rule $p / n \rightarrow I$ in the routing table of $r$ for any $I$, nor any rule $p^{\prime} / n^{\prime} \rightarrow I$ where $p^{\prime}$ is a prefix of $p$. The implications of this constraint largely depends on $p$, therefore on the adresses of $m_{0}$ and $m_{1}$.

\section{ROUTING TABLE INFERENCE}

The constraints retained from observation in III-B don't directly provide an estimate of the routing table. Many routing table are compatible with these constraints. However, combining the constraints with additional assumptions allows us to infer realistic rules. We will examine three inference patterns, using different assumptions to infer the routing table of a router.

\section{A. Most specific routing table}

The most simple inference pattern simply translates the Existence constraint from Section III-B into rules, using the trivial prefix $m / 32$ for each monitor $m: m / 32 \rightarrow m(t)$. We then merge duplicate rules as described in Section III-A(2). The Consistence constraint is trivially ensured, since each rule is either of prefix-length 32 or resulting from a duplicate merge.

This routing table is rigorously consistent with the observation, and makes no additional assumption at all. However, its 
reach is very limited, since it only provides routing information towards destination inside our monitor set. We name this infered table the most specific routing table.

\section{B. Generalizing hypotheses}

At the extreme opposite of the most specific routing table (Section IV-A), there is another routing table that is compatible with the observation: the least specific routing table. It consists on the set of rules with the largest sets of destinations (or the shortest prefixes) that are compatible with the Consistence constraint from Section III-B. While this routing table is very general, however, it is very hard to ensure its completedness: one may find a destination $d$ which, if added to $M$, would produce incompatible rules. For example, if $M$ is a single monitor $m_{1}$ such that $m_{1}(t)=\left\{i_{1}\right\}$, then the least specific routing table consists in only one rule, $\left\{\emptyset / 0 \rightarrow\left\{i_{1}\right\}\right\}$ ("empty prefix routes using $i_{1}$ "). If there exists a host $m_{2}$ such that $m_{2}(t) \neq\left\{i_{1}\right\}$, then adding $m_{2}$ to the monitor set makes the routing table incompatible. Note that, the larger and the better distributed $M$ is, the harder it is to find destinations that are not compatible with the routing table, thus the more relevant the least specific routing table is.

The actual routing table of a router is somewhere between the most specific ("least informative but most accurate") and the least specific ("most informative but least accurate") routing table. Using well-chosen generalizing hypotheses, we can extend the rules infered from the Existence constraint. Such a generalizing hypothesis consists in an assumption on the structure of the prefixes in the ruleset. It can be elaborated by leveraging knowledge on the networks, such as practical constraints or common implementations. In addition to the least specific routing table in Section IV-C, we will discuss one such generalizing hypothesis based on AS prefixes in Section IV-D.

\section{CIDR prefixes generalization}

The least specific, most generalized routing table is actually a generalizing hypothesis resulting from the CIDR convention. For many reasons, among which the practical size of the routing tables, the CIDR address allocation method [5], [6], [7] was introduced in 1993 and is now a both formal and practical standard. The adoption of CIDR means that subnetworks are characterized by address prefixes. This allows for efficient routing table compression, since the rules can be expressed in the form of prefix-matching rules, both easy to lookup using dedicated hardware [8] and of small size compared the classful rules of the early Internet. From an inference point of view, this means that each prefix-based rule only needs one representant to be discovered. The least specific routing table is the routing table in which the prefixes are as small as possible while remaining compatible with the observation. Algorithm 1 is designed to construct this table efficiently.

1) Inference algorithm: $I$ is an associative map indexed with the monitor adresses that contains the set of observed interfaces for a given target when responding to each monitor, i.e. $I[m]=m(t)$, and $I[a, b]$ designates the list of $I[k]$ for

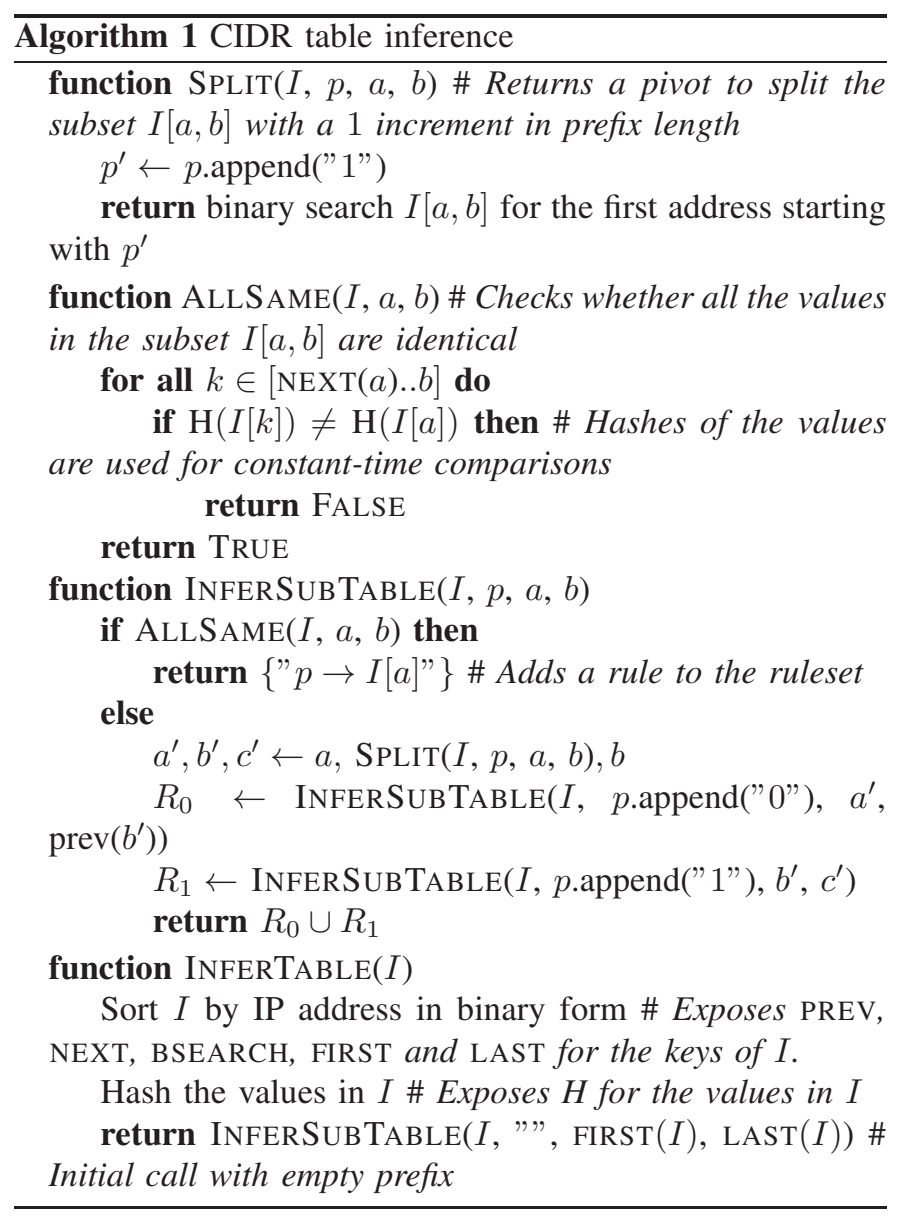

$a \leq k \leq b$. The algorithm first sorts $I$ by keys so that it can perform a fast binary search of prefixes cuts. The main recursive function returns, for a given binary prefix $p$ and a contiguous subset of $I$ (described by its boundary keys $a$ and $b$ ), the set of rules required to be consistent with the data containing the least number of rules with the shortest (most general) prefixes. To do so, it recursively calls itself with increasingly long prefixes, stopping when the subset is either empty or all its elements observe the same interface set (indicating that they can be grouped under a single rule). If at least two elements of the subset require different rules, then the prefix length is increased to further differentiate the subsets.

2) Proof and speed of the algorithm: Algorithm 1 consists in one entry routine and three subroutines.

The subroutine $\operatorname{SpLit}(I, p, a, b)$ takes 4 arguments: $I$ is a lexically key-sorted dictionary, containing key-values pairs. Each key is a monitor identifier (its IP address) and each value is the set of the interfaces observed by this monitor for the given target. $p$ is a binary prefix, represented by a byte row. $a$ and $b$ are monitor addresses. It is assumed that the subset $[a, b]$ of keys lexically comprised between $a$ and $b$ share a common prefix $p$. SPLIT returns the first (in lexical order) monitor address $x$ such that all the keys $x_{0}<x$ match the prefix $p .0$ and all the keys $x_{1} \geq x$ match the prefix $p .1$. Since 
$I$ is lexically sorted, then $[a, b]$ is lexically sorted too and a binary search allows to find $x$ in $O(\lg (|[a, b]|))$.

The subroutine $\operatorname{All} \operatorname{Same}(I, a, b)$ takes 3 arguments defining a subdictionary $I[a, b]$ of sets of observed interfaces indexed by observing monitor. AlLSAmE tests whether all the elements $I[x]$ for $x \in[a, b]$ are equal. This is achieved by comparing each $I[x]$ for $a<x \leq b$ to $I[a]$. If at least one element doesn't match, the routine returns FALSE. Otherwise, it returns TRUE. By pre-hashing the values of each $I[x]$, the equality test is performed in $O(1)$, and the full execution of the routine completes in $O(|[a, b]|)$.

The core subroutine InferSubTable $(I, p, a, b)$ takes 4 arguments, under the same restrictions as the arguments of SPLIT. It returns a list $R$ of rules in the form $p_{k} \rightarrow I_{k}$ such that:

- $R$ is consistent with the observation, i.e if $x$ matches $p_{k}$ then $I[x]=I_{k}$.

- $R$ is minimum, i.e. there exists no ruleset $R^{\prime}$ such that $\left|R^{\prime}\right|<|R|$ and $R^{\prime}$ is consistent with the observation.

This can be proven by recurrence on the value of $n=P_{\max }-$ $|p|$ where $P_{\max }=32$ is the maximum prefix length.

If $n=0$, then $p$ is a full-length prefix, actually matching exactly one address, $a=b$. Then $L=\{p=a \rightarrow I[a]=I[b]\}$ is the minimal solution.

If $n>0$, then there are two cases.

- All the elements in $I[a, b]$ are equal, in which case $L=$ $\{p \rightarrow I[a]=I[b]=I[x]\}$ for any $x \in[a, b]$ is the minimal consistent solution.

- There are at least two elements in $I[a, b]$ that are not equal, say $x$ and $y . x$ and $y$ have atleast one different bit, proving that $p$ is not specific enough. $p$ is further specified by appending one bit to the pattern, either 0 or 1 , using the least-specific split computed by SPLIT and calling recursively calling the subroutine with a prefixlength of $|p|+1$. By recurrence, the two sub-solutions are optimal, and therefore the union of the two solutions are also optimal, since there exist no solution with a prefixlength of $|p|$.

The subroutine internal calculations are: the call to the subroutine AlLSAME, executing in $O(|[a, b]|)$, the call to the subroutine SPLIT, executing in $O(\lg (|[a, b]|))$, and finally the recursive calls. An amortized analysis shows that for each prefix length $l^{\prime} \leq|p|$, each element $I[x]$ is only looped trough once, therefore bounding the complexity to $O\left(|[a, b]| *\left(P_{\max }-\right.\right.$ $|p|)$ ) where $P_{\max }$ is the maximum prefix length (32).

The main routine INFERTABLE $(I)$ takes only one parameter representing the observed data. It returns the minimum CIDR ruleset consistent with the observation. It firsts builds the required representation to fit the assumptions above, in particular that $I$ is a sorted dictionary allowing for efficient binary searches and a proper chaining allowing the usage of NEXT and PREV on the keys of $I$. The values of $I$ are also hashed to expose a constant-time equality test through the memoized $\mathrm{H}$ hash function. It then calls the INFERSUBTABLE subroutine with the initialization parameters: $a$ is the first key of $I, b$ is the last key of $I$, and the prefix is initially empty, satisfying the required constraints. This main entry routine performs timeconsuming pre-processing. The sorting of the keys of $I$ is $O(|M| \times \ln (|M|))$ where $M$ is the number of monitors, i.e. the number of keys in $I$. The hashing can also be achievement in $O(|M|)$ since the size of the hashed sets is bounded and low (no more than a few dozens elements in the worst cases) allowing for a very efficient binary hashing, regardless of the specific implementation of the hashing function - any efficient generic binary hashing function will work. Last but not least, the execution of the unique call to INFERSUBTABLE has a time complexity of $O\left(|M| * P_{\max }\right)$. The speed of the algorithm has a total complexity of $O(|M| \times \lg (|M|))$, but has significant hidden constants, in particular for the non-dominant terms of the complexity formula $\left(P_{\max }=32\right.$ and hash function calculations hidden constants).

\section{AS prefixes generalization}

The above method is the most generalizing, least specific hypothesis that is consistent with the observation and in which destination classes $D_{k}$ match destination prefixes. However, this assumption doesn't seem realistic, since it can infer very short prefixes (i.e. very general rules) based on the sparse nature of the monitor set. To avoid too general assumption, we restrict the selected prefixes to the prefixes advertised by ASes.

To do so, we use an algorithm very close to Algorithm 1. To account for the restriction to AS prefixes, instead of stopping the recursion whenever a subset of monitors observe the same interface set, we continue until the prefix is a prefix claimed by an AS, or the monitor set only has one element (prefix length is 32), to ensure that each target is in the output table. This can be done efficiently by looking up the prefix in a binary search tree, based upon an official prefix registry, such as Routeviews [9].

\section{Measurement}

To assess the feasibility and the relevance of our approach, we have conducted a practical measurement of Distributed UDP Ping and then performed the routing table informance method described above.

\section{A. Repeated Distributed UDP Ping}

The repeated Distributed UDP Ping was realized from the PlanetLab platform, consisting of 548 monitors distributed among 193 ASes. 2276 targets were chosen among routers responding to UDP Ping probes from a previous experiment [1]. The measure consisted in 30 repeated Distributed UDP Ping measurement towards each target spanning over about 10 minutes.

We combined the output of the repeated measurements for each target, and for each target, we compute its table using the methods desbribed earlier: the most specific table, the CIDR prefixes tables, and the AS prefixes tables. 


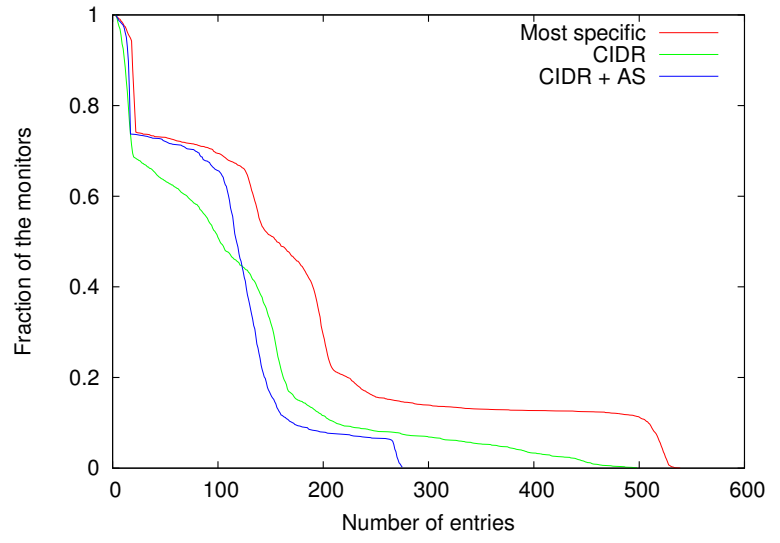

Fig. 3. Inverse cumulative distribution of the number of entries using three refinement methods: most specific, CIDR prefixes, and AS prefixes.

\section{B. Impact of the inference method}

After processing the observation from Distributed UDP Ping into Existence and Consistence constraints, we used the three inference patterns describes in Section IV to compute estimates of the routing tables for the target routers. For each inference pattern and for each target, we obtain a list of rules consistent with the constraints, composed of pairs of a prefix and a list of interfaces used to respond to monitors matching these prefixes.

We then computed the number of rules obtained for each target with the three inference methods (See Fig. 3). Intuitively, for a given observation, less rules means more efficient routing table, since the CPU and memory required to perform the routing depend on the number of rules in the table.

The most specific routing tables have a higher number of entries, since there is one entry per monitor which are able to observe each target. Using AS-advertised prefixes requires less rules in the worst cases (when the most high number of rules is required) but using the shortest CIDR prefixes performs best for simpler tables. This suggests that in practice, either of the two methods may be used, or mixed, to provide the most efficient results.

\section{Impact of the number of monitors}

We have suggested in Sections II and III that the nature of the monitor set can widely affect the nature of the observation and of the constraints. To assess the extend of this phenomenon, we have emulated different monitor sizes by filtering the data to only keep the results from random subsets (of given size, lower than the maximum number of available monitors) of our complete monitor set, and comparing the results. (See Fig. 4, 5, 6)

We observe that the amplitude of the distribution depends a lot on the number of monitors, suggesting that even if colocation is captured by the CIDR prefixes based methods, the monitor set has not reached a steady size and could be improved. However, the shape of the distribution remains consistent with the monitor size, suggesting that adding monitors may give more precise, but not completely different results.

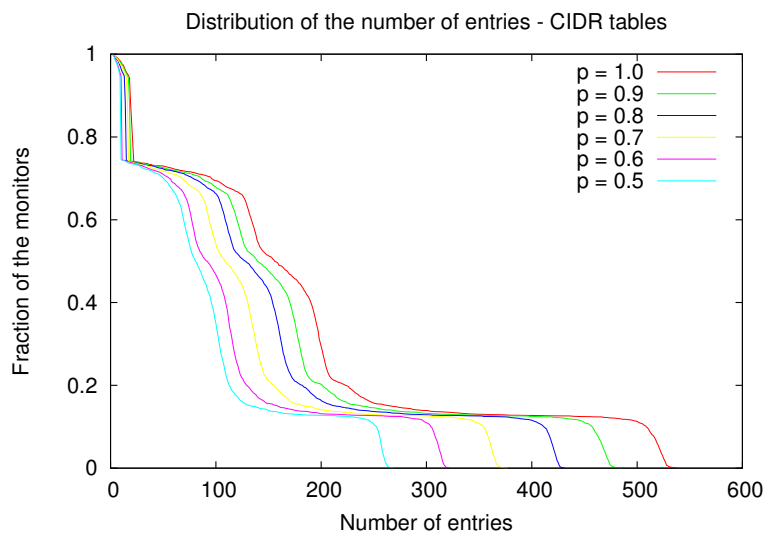

Fig. 4. Inverse cumulative distribution of the number of entries in the most specific table for several monitors subset sizes.

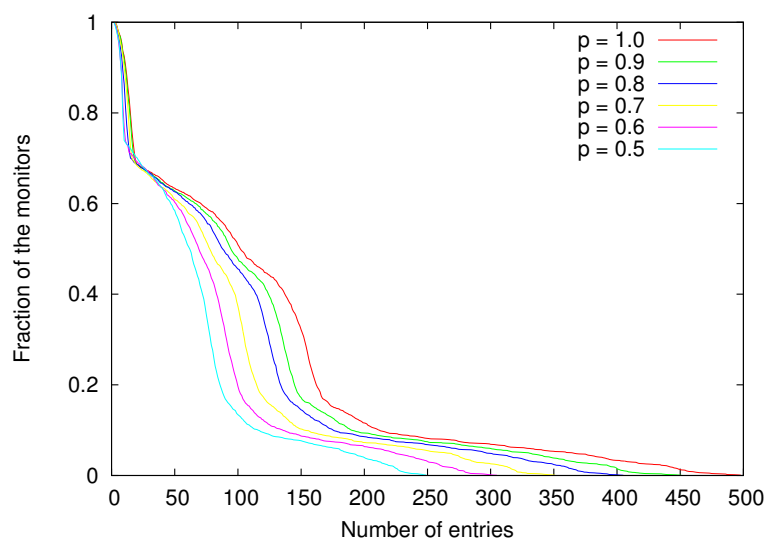

Fig. 5. Inverse cumulative distribution of the number of entries in the CIDR prefixes table for several monitors subset sizes.

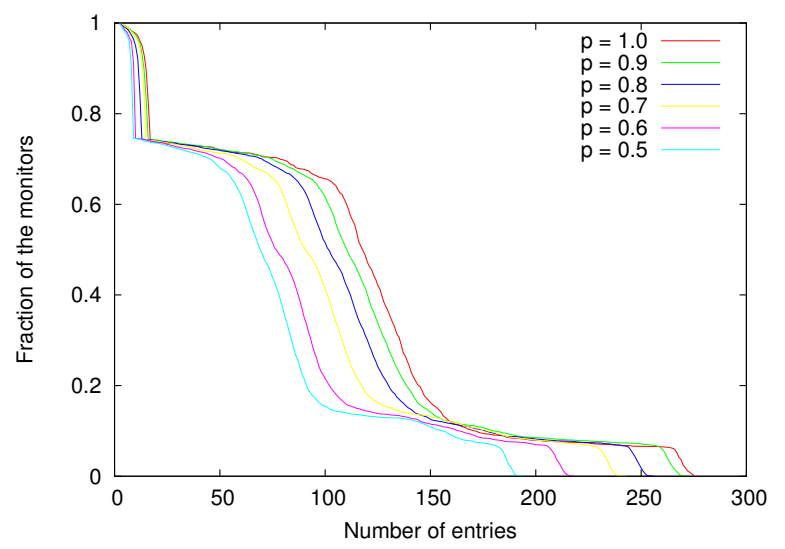

Fig. 6. Inverse cumulative distribution of the number of entries in the AS prefixes table for several monitors subset sizes.

\section{RELATED WORK}

The physical and IP-level internet topologies are extensively studied since the seminal papers of Pansiot et al. [10] and Faloutsos et al. [11]. The most classical approach consists in building maps from traceroute-like measurements. However, several studies have shown that obtained maps are intrinsically 
biased [12], [13], [14], and even that traceroute outputs are unreliable [15], [16], [17]. The hope that increasing the size and quality of maps would overcome these issues has led to much effort, but the situation remains far from satisfactory [14], [18], [19].

Conducting precise measurements of random nodes to obtain a reliable estimate of their behaviour was first suggested in [12]. We explored the possibility to do so at IP level in [4] but we only partly succeeded and we conducted thorought simulations in [20].

Our work is also closely related to alias resolution (which plays a key role in the building of maps): while we seek all (unknown) interfaces of a given router identified by one of its interfaces, alias resolution aims at identifying in a given set of interfaces the ones that belong to a same router [21], [22]. Probes similar to ours are used in this context, in particular by the iffinder tool [23], as well as other techniques. Our use of such probes was clearly inspired by these works.

Finally, important efforts are devoted to the deployment of large and distributed measurements infrastructures, which are crucial for this field of research [24], [25], [26], [27], [28]. Some of them distribute monitoring capabilities at a huge scale (typically onto thousands of end-hosts) and so are particularily promising for us [28], [25].

\section{CONCLUSION}

In this work, we have exposed the principle of using a distributed UDP Ping measurement to gain insight on the routing tables of the measured targets. However, the relevance of the estimate relies highly on the quality of the monitor set, since the inference methods only allows to generalize rules in adress scopes (subnetworks) in which there are monitor from the monitor set.

Besides the improvement of the monitor set, several factors could be utilized to further infer the rules: implementation details of the routing algorithms (namely BGP and OSPF) at the subnetwork, area and AS level, default routes, and the usage of looking glasses. The repetition over time of this measurement and inference method may be used to track the routing dynamics of a given target, in particular after a BGP update.

Acknowledgements. This work is partly supported by the European Commission FP7 EULER project (grant 258307), Future Internet Research and Experimentation (FIRE), and by the DynGraph grant ANR-10-JCJC-0202 from the Agence Nationale de la Recherche.

\section{REFERENCES}

[1] M. Latapy, C. Crespelle, E. Rotenberg, and F. Tarissan, "Measuring the degree distribution of routers in the core internet," submitted.

[2] R. Braden, "Requirements for Internet Hosts - Communication Layers," RFC 1122 (INTERNET STANDARD), Internet Engineering Task Force, Oct. 1989, updated by RFCs 1349, 4379, 5884, 6093, 6298, 6633, 6864. [Online]. Available: http://www.ietf.org/rfc/rfc1122.txt

[3] F. Baker, "Requirements for IP Version 4 Routers," RFC 1812 (Proposed Standard), Internet Engineering Task Force, Jun. 1995, updated by RFCs 2644, 6633. [Online]. Available: http://www.ietf.org/rfc/rfc1812.txt
[4] C. Crespelle, M. Latapy, and É. Rotenberg, "Rigorous measurement of ip-level neighborhood of internet core routers," in 2nd IEEE International Workshop on Network Science and Communication Networks (NetSciCom'10), 2010.

[5] Y. Rekhter and T. Li, "An Architecture for IP Address Allocation with CIDR," RFC 1518 (Historic), Internet Engineering Task Force, Sep. 1993. [Online]. Available: http://www.ietf.org/rfc/rfc1518.txt

[6] V. Fuller, T. Li, J. Yu, and K. Varadhan, "Classless InterDomain Routing (CIDR): an Address Assignment and Aggregation Strategy," RFC 1519 (Proposed Standard), Internet Engineering Task Force, Sep. 1993, obsoleted by RFC 4632. [Online]. Available: http://www.ietf.org/rfc/rfc1519.txt

[7] V. Fuller and T. Li, "Classless Inter-domain Routing (CIDR): The Internet Address Assignment and Aggregation Plan," RFC 4632 (Best Current Practice), Internet Engineering Task Force, Aug. 2006. [Online]. Available: http://www.ietf.org/rfc/rfc4632.txt

[8] A. J. Mcauley and P. Francis, "Fast routing table lookup using cams," in IEEE INFOCOM, 1993, pp. 1382-1391.

[9] A. Broido and k. claffy, "Analysis of RouteViews BGP data: policy atoms," in Network Resource Data Management Workshop, Santa Barbara, CA, May 2001.

[10] J.-J. Pansiot and D. Grad, "On routes and multicast trees in the internet," SIGCOMM Comput. Commun. Rev., vol. 28, no. 1, pp. 41-50, 1998.

[11] M. Faloutsos, P. Faloutsos, and C. Faloutsos, "On power-law relationships of the internet topology," in SIGCOMM, 1999, pp. 251-262.

[12] A. Lakhina, J. W. Byers, M. Crovella, and P. Xie, "Sampling biases in ip topology measurements," in INFOCOM, 2003, pp. 332-341.

[13] D. Achlioptas, A. Clauset, D. Kempe, and C. Moore, "On the bias of traceroute sampling: or, power-law degree distributions in regular graphs," J. ACM, vol. 56, no. 4, 2009

[14] W. Willinger, D. Alderson, and J. C. Doyle, "Mathematics and the internet: A source of enormous confusion and great potential," Notices of the AMS, vol. 56, no. 5, pp. 586-599, May 2009.

[15] F. Viger, B. Augustin, X. Cuvellier, C. Magnien, M. Latapy, T. Friedman, and R. Teixeira, "Detection, understanding, and prevention of traceroute measurement artifacts," Computer Networks, vol. 52, no. 5, pp. 9981018, 2008.

[16] B. Donnet, M. Luckie, P. Mérindol, and J. Pansiot, "Revealing MPLS tunnels obscured from traceroute," ACM SIGCOMM Computer Communication Review (CCR), vol. 42, no. 2, pp. 87-93, Apr 2012.

[17] M. Roughan, W. Willinger, O. Maennel, D. Perouli, and R. Bush, "10 lessons from 10 years of measuring and modeling the internet's autonomous systems," Selected Areas in Communications, IEEE Journal on, vol. 29, no. 9, pp. 1810-1821, 2011.

[18] P. Barford, A. Bestavros, J. W. Byers, and M. Crovella, "On the marginal utility of network topology measurements," in Internet Measurement Workshop, 2001, pp. 5-17.

[19] M. Latapy and C. Magnien, "Complex network measurements: Estimating the relevance of observed properties," in INFOCOM, 2008, pp. 1660-1668.

[20] C. Crespelle and F. Tarissan, "Evaluation of a new method for measuring the internet degree distribution: Simulation results," Computer Communications, vol. 34, no. 5, pp. 635-648, 2011.

[21] M. Gunes and K. Sarac, "Importance of ip alias resolution in sampling internet topologies," in IEEE Global Internet Symposium, 2007, 2007, pp. 19-24.

[22] K. Keys, "Internet-scale ip alias resolution techniques," ACM SIGCOMM Computer Communication Review, vol. 40, no. 1, pp. 50-55, 2010.

[23] B. Huffaker, D. Plummer, D. Moore, and K. Claffy, "Topology discovery by active probing," in Applications and the Internet (SAINT) Workshops, 2002. Proceedings. 2002 Symposium on, 2002, pp. 90-96.

[24] CAIDA, "Caida, macroscopic topology measurement projects," 2010, http://www.caida.org/projects/macroscopic/.

[25] Y. Shavitt and E. Shir, "Dimes: let the internet measure itself," Computer Communication Review, vol. 35, no. 5, pp. 71-74, 2005.

[26] H. V. Madhyastha, T. Isdal, M. Piatek, C. Dixon, T. Anderson, A. Krishnamurthy, and A. Venkataramani, "iplane: An information plane for distributed services," in In OSDI 2006, 2005.

[27] P. Consortium, "Planetlab: An open platform for developing, deploying and accessing planetary-scale services," 2009, http://www.planet-lab. org/.

[28] RIPE-NCC, "Ripe atlas," https://atlas.ripte.net. 